Casos Clínicos

Arch. Esp. Urol., 59, 7 (725-727), 2006

\section{ORQUITIS IDIOPÁTICA GRANULOMATOSA: ESTUDIO ANATOMOPATOLÓGICO DE UN CASO.}

Miguel Martínez-Rodríguez', Samuel Navarro Fos', Pilar Soriano Sarriól, Carlos Alcalá-Santaella Casanova², David Ramos Soler' y Antonio LlombartBosch?

Departamento de Anatomía Patológica'. Servicio de Urología ${ }^{2}$ del Hospital Clínico Universitario de Valencia. Valencia. España.

Resumen.- OBJETIVO: Presentar un caso de orquitis granulomatosa idiopática, patología extremadamente infrecuente, en un paciente de 76 años.

MÉTODOS/RESULTADOS: Se recibe testículo derecho con diagnóstico clínico y ecográfico de tumor testicular. En el estudio anatomopatológico se observa la ausencia de celularidad neoplásica y se aprecia hallazgos morfológicos compatibles con orquitis granulomatosa idiopática.

CONCLUSIONES: La orquitis idiopática granulomatosa es una entidad de etiología desconocida, ecográfica y clínicamente no distinguible de una neoplasia testicular, llegándose al diagnóstico tras la orquiectomía.

Palabras clave: Orquitis idiopática granulomatosa. Aspecto ecográfico tumoral. Mecanismo autoinmune. Orquiectomía.

Miguel Martínez Rodríguez Servicio de Anatomía Patológica Hospital Clínico Universitario de Valencia Avda. Vicente Blasco lbáñez, 17 46010 Valencia. (España) miguelmartinez@comv.es

Trabajo recibido: 21 de noviembre 2005
Summary.- OBJECTIVE: To report one case of idiopathic granulomatous orchitis, an extremely rare disease, in a 76yearold patient.

METHODS/RESULTS: The pathology department received a testicle with the clinical/radiological diagnosis of testicular tumor. The pathologic study showed absence of neoplasias and presence of morphological findings compatible with idiopathic granulomatous orchitis.

CONCLUSIONS: The idiopathic granulomatous orchitis is an entity of unknown etiology, clinically or ultrasonographically not distinguishable from testicular neoplasias, the diagnosis of which is made after orchiectomy.

Keywords: Idiopathic granulomatous orchitis. Tumor-like ultrasound features. Autoimmune mechanism. Orchyectomy.

\section{INTRODUCCIÓN}

La orquitis granulomatosa testicular engloba a un grupo heterogéneo de patologías entre las que se encuentran las de causa conocida, atribuídas principalmente a Mycobacterium tuberculosis y a otras menos frecuentes como la brucelosis, actinomicosis y asociada a sarcoidosis, (1) y las de causa desconocida o idiopática (OGI). Ésta última supone una entidad rara e inusual, descrita en 1926 por Grünberg, quién la definió como una lesión inflamatoria crónica e inespecífica caracterizada por un cuadro histológico consistente en la aparición de lesiones granulomatosas, con presencia de numerosas células gigantes multinucleadas y ausencia de necrosis caseosa (2).

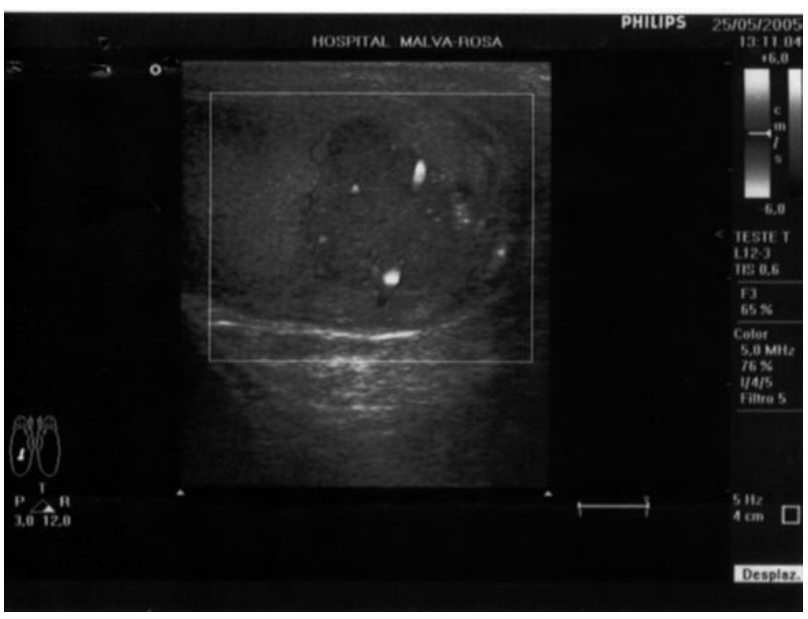

FIGURA 1. Masa sólida e irregular testicular hipoecoica. 


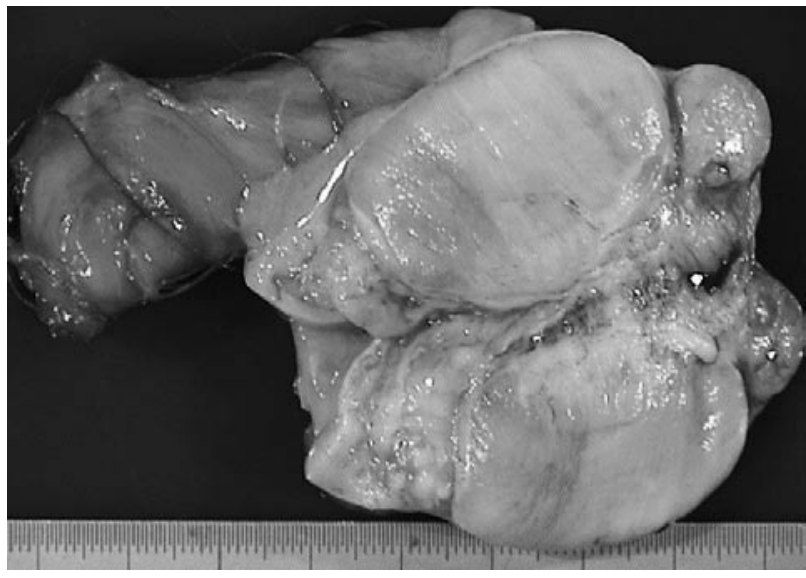

FIGURA 2. Testículo seccionado mostrando aspecto fibroso sin evidencia de lesión neoplásica.

Clínica y ecográficamente se presenta como una neoplasia testicular, y su diagnóstico se realiza post-orquiectomía en la práctica totalidad de casos (3-4). Su etiología actualmente es desconocida, existiendo hasta el momento varias teorías que intentan explicar el proceso: la vascular, por arteritis de los vasos testiculares (2), la traumática y la inmunológica (2).

\section{CASO CLÍNICO}

Se presenta el caso de un paciente de 76 años de edad, sin alergias conocidas, con antecedentes de dislipemia, hipertensión arterial, infarto agudo de miocardio anti- guo, triple derivación aorto-coronaria por enfermedad de los 3 vasos de un año de antigüedad y RTU por hiperplasia leiomioadenomatosa de próstata de dos años de antigüedad. No se refiere ningún traumatismo testicular previo. Tampoco presenta antecedentes clínicos, radiológicos ni analíticos de tuberculosis ni brucelosis. Acude a la consulta de urología por molestias testiculares, consistentes en dolor leve crónico intermitente, de 4 meses de evolución, apreciándose a la exploración nódulo duro e irregular en testículo derecho sin dolor acompañante espontáneo ni a la palpación.

Los hallazgos ecográficos revelan una lesión focal nodular, lobulada y de baja ecogenicidad de $2 \mathrm{~cm}$ de diámetro en polo inferior del testículo derecho, vascularizada, siendo, por tanto compatible con neoformación (Figura 1). Testículo izquierdo sin hallazgos patológicos de interés.

En la TAC abdómino pélvico con contraste no se evidencian adenopatías retroperitoneales.

El análisis sanguíneo muestra valores dentro de la normalidad para alfa-feto proteína, HCG y LDH (HCG: 1,74 U/l; Alfa-feto proteína: $2.07 \mathrm{ng} / \mathrm{ml}$; LDH: 299 $\mathrm{mU} / \mathrm{ml}$ ).

El paciente es sometido a una orquiectomía inguinal derecha.

El estudio anatomopatológico macroscópico muestra bolsa escrotal con hidrocele de $8 \times 5 \times 2,5 \mathrm{~cm}$ con 5 $\mathrm{cm}$ de conducto epididimario. A la sección se observa
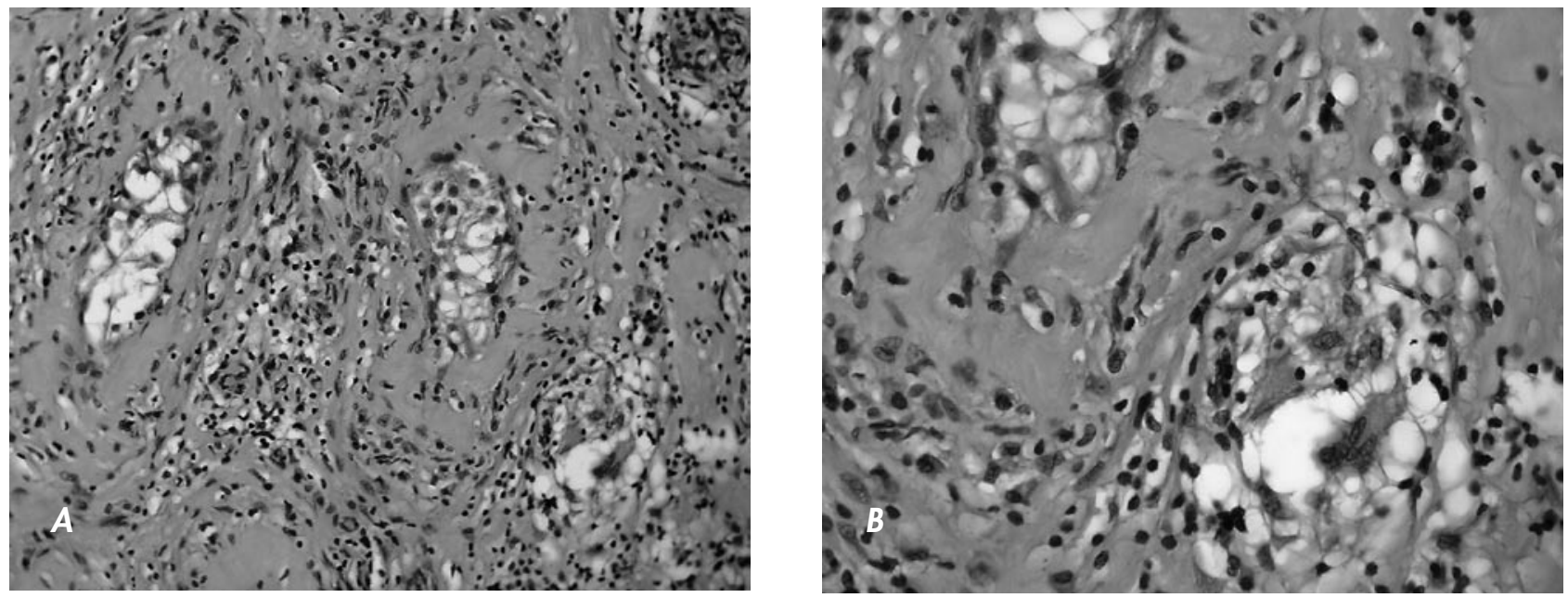

FIGURA 3. A) Examen microscópico mostrando infiltrado inflamatorio intersticial con formación granulomatosa y con infiltrado linfoplasmocitario tubular (H\&E, 10x).

B) Detalle de la lesión granulomatosa mostrando una célula multinucleada gigante en el cuadrante inferior derecho (H\&E, 40x). 
testículo de 5,5 ×2,5 ×2,5 cm, de consistencia fibrosa, sin lesiones macroscópicas compatibles con neoplasia (Figura 2). El estudio microscópico muestra parénquima testicular con degeneración vacuolada de células germinales y de Sertoli, con núcleo vesicular, apreciándose en las paredes de los túbulos seminíferos un infiltrado inflamatorio mononuclear con linfocitos y células plasmáticas. Se distinguen numerosas formaciones granulomatosas con abundantes células multinucleadas gigantes ubicadas en el estroma entre los túbulos seminíferos $y$, alguna de ellas, en el interior de los mismos. No se observa celularidad neoplásica (Figura $3 \mathrm{~A}$ y $3 \mathrm{~B}$ ). Al efectuarse las tinciones de PAS y Ziehl Nielsen no se observan microorganismos micóticos ni micobacterias. Los hallazgos histopatológicos sugieren el diagnóstico de orquitis idiopática granulomatosa.

\section{DISCUSIÓN}

Las enfermedades granulomatosas testiculares incluyen a aquellas originadas por agentes etiológicos específicos y la forma idiopática. Dentro del primer grupo, los agentes causales más frecuentes son el bacilo tuberculoso y la brucelosis, siendo los testículos el órgano del aparato urinario más inusualmente afectados en la tuberculosis y la localización más tardía.(5)

La mayoría de los casos de orquitis granulomatosa idiopática se diagnostican entre la sexta y la séptima décadas de vida, presentándose clínicamente como dolor leve testicular, hinchazón y fiebre ocasional. En algunos casos, como el que se describe en este estudio, solamente aparece masa testicular asociada a dolor crónico de escasa intensidad.

La etiología de la OGI es desconocida, pero se ha asociado con antecedentes de traumatismo y cirugía testicular previa. Los granulomas observados después de un traumatismo testicular remedan a los observados en la OGI. Russell y Friedman consiguieron reproducir experimentalmente en animales lesiones similares cortando los vasos deferentes e implantándolos en la piel del escroto (6).

En 1954 Berg aisló de la cabeza de los espermatozoides una fracción lipídica similar a la aislada en el bacilo tuberculoso, presente en todos los cortes histológicos de orquitis granulomatosa tuberculosa (7). Actualmente se cree que un importante traumatismo testicular podría generar una salida de los túbulos de los espermatozoides y esto originaría una reacción autoinmune que desencadenaría el proceso (3-2).
El diagnóstico de la $\mathrm{OGI}$ supone un proceso complejo. La ecografía escrotal es una técnica de imagen muy sensible para el diagnóstico de patología testicular focal y difusa. Se ha descrito un patrón ecográfico de textura heterogénea para neoplasias y homogéneo en patología inflamatoria. El engrosamiento del epidídimo, piel escrotal y túnica albugínea se consideran indicadores de procesos inflamatorios benignos (1). No obstante, al ser la OGI una lesión intratesticular en la que se produce destrucción de la arquitectura tisular con fibrosis, ecográficamente es una lesión indistinguible de los tumores de células germinales y linfomas (5).

Dada la imposibilidad de diagnóstico diferencial entre la OGI y la patología neoplásica testicular, el diagnóstico de OGI se alcanza, en la práctica totalidad de ocasiones, con el estudio anatomopatológico del testículo tras orquiectomía, siendo también éste el único tratamiento eficaz que resuelve los síntomas (2).

\section{BIBLIOGRAFÍA y LECTURAS RECOMENDADAS ( ${ }^{*}$ lectura de interés $y^{* *}$ lectura fundamental)}

*1. SALMERÓN, I.; RAMÍREZ-ESCOBAR, M.; PUERTAS, F. y cols.: "Granulomatous epididymoorchitis: sonographic features and clinical outcome in brucellosis, tuberculosis and idiopathic granulomatous epididymo-orchitis". J. Urol., 159: 1954, 1998.

**2. OSCA GARCÍA, J.M.; ALFARO FERRERES, L.; RUIZ CERDA, J.L. y cols.: "Orquitis granulomatosa idiopática”. Actas Urológicas Españolas, 17: 53, 1993.

**3. KAHN, R.I.; MCANINCH, J.W.: "Granulomatous disease of the testis". J. Urol., 123: 868, 1980.

*4. MOGENSEN, M.; NINO-MURCIA, M.: "Idiopathic granulomatous epididymo-orchitis". J.Ultrasound Med., 24: 1007, 2005.

5. KLEIN, F.A.; VICK, C.W.; SCHNEIDER, V.: "Bilateral granulomatous orchitis: manifestation of idiopathic sistemic granulomatosis". J. Urol., 134: 762, 1985.

6. RUSSELL, M.; FRIEDMAN, N.B.: "Studies in general byology of sperm: experimental production of spermatic granuloma”. J. Urol., 65:650, 1951.

7. BERG, J.W.: "An acid-fast lipid from spermatozoa". Arch. Path., 57: 115, 1954.

8. AITCHINSON, M.; MUFTI, G.R.; FARRELL, J. y cols.: "Granulomatous orchitis: review of 15 cases". Brit. J. Urol., 66: 312, 1990. 